\title{
Extracting Hand Grasp \& Motion for Intent Expression in Mid-Air Shape Deformation : A Concrete \& Iterative Exploration through a Virtual Pottery Application
}

\author{
Vinayak $^{\mathrm{a}}$, Karthik Ramani ${ }^{\mathrm{a}, \mathrm{b}}$ \\ ${ }^{a}$ School of Mechanical Engineering \\ Purdue University \\ ${ }^{b}$ School of Electrical and Computer Engineering \\ Purdue University (by courtesy)
}

\begin{abstract}
We describe the iterative design and evaluation of a geometric interaction technique for bare-hand mid-air virtual pottery. We model the shaping of a pot as a gradual and progressive convergence of the pot-profile to the shape of the user's hand represented as a pointcloud (PCL). Our pottery-inspired application served as a platform for systematically revealing how users use their hands to express the intent of deformation during a pot shaping process. Our approach involved three stages: (a) clutching by proximal-attraction, (b) shaping by proximal-attraction, and (c) shaping by grasp+motion. The design and implementation of each stage was informed by user evaluations of the previous stage. Our work evidently demonstrates that it is possible to enable users to express their intent for shape deformation without the need for a fixed set of gestures for clutching and deforming a shape. We found that the expressive capability of hand articulation can be effectively harnessed for controllable shaping by organizing the deformation process in broad classes of intended operations such as pulling, pushing, and fairing. After minimal practice with the pottery application, users could figure out their own strategy for reaching, grasping, and deforming the pot. Users particularly enjoyed using day-to-day physical objects as tools for shaping pots.
\end{abstract}

Keywords: Mid-air gestures, depth sensor, virtual pottery, shape deformation, hand grasp.

\section{Introduction}

2 Mid-air gestures have been widely used as the symbolic 3 means for expressing user's intent in 3D shape modeling [1, $\left.{ }_{4} 2,3,4,5,6\right]$. Gesture-based interactions enable the user to fo5 cus on the design task rather than dedicating significant time 6 towards learning the usage of the tool itself [7]. With the re7 cent commercialization of depth cameras, gesture-based inter8 actions have become accessible to the common user; creative 9 applications for free-form shape modeling [8] in mid-air have 10 gained significant popularity. The user input in these applica11 tions is represented as a combination of some special hand pos12 ture (such as pointing with a finger), and the motion of a repre13 sentative point (such as the palm or finger-tip) on the hand.

${ }_{14}$ Hand and finger movements in real-world shaping processes 15 (such as pottery or clay sculpting) are complex, iterative, and ${ }_{16}$ gradual. Such processes are essentially governed by the physics 17 and geometry of contact between the hand and clay. Thus, the 18 true expressive potential of finger movements remains under19 utilized despite advances in hand pose and skeletal estimation [9, 20 10]. This is what drives our research wherein, our intention is ${ }_{21}$ to bridge the gap between the user's expression of intent and 22 the corresponding deformation of a virtual shape.

In this paper, we give an comprehensive account of our re24 cent works $[11,12]$ by describing the iterative design and evalu25 ation of a geometric interaction technique for bare-hand mid-air 26 virtual pottery. Our broader goals are to (a) identify aspects of
27 real-world interactions that can be emulated in free-form $3 \mathrm{D}$ 28 shape deformation, (b) understand the expression of design in29 tent in shape deformation in terms of the user's hand grasp and 30 motion, and (c) design an interaction that integrates the geo31 metric information in user's actions with shaping operations in 32 virtual space.

\section{1.1. Contributions}

34 This paper is an extension of our recent work [12], where 35 we modeled the shaping of a pot as a gradual and progressive 36 convergence of the pot's profile to the shape of the user's hand 37 represented as a point-cloud (PCL). We presented a method that 38 uses the kernel-density estimate (KDE) of the hand's PCL to ex39 tract the grasp and motion for deforming the shape of a pot in ${ }_{40} 3 \mathrm{D}$ space. This feature of our method directly allows a user to ${ }_{41}$ shape pots by using physical artifacts as tools without the need 42 for computing any finite set of gestures or hand skeleton. In 43 doing so, we demonstrate that it is possible to achieve control44 lability in bare-hand mid-air shape deformation using raw PCL 45 data of the user's hand.

46 There are two differences between this paper and our prior 47 works $[11,12]$. First, we present the complete evolution of our 48 algorithm in three stages of iterative design (section 3.3). At ${ }_{49}$ the end of each stage, we describe a user evaluation that in50 forms the algorithm development of the subsequent stage. Sec51 ond, we evaluate our KDE based approach in comparison to our 52 prior work [11]. Our evaluations help reveal two core aspects 
53 of mid-air interactions for shape deformation, namely, intent \& 54 controllability. We characterize user behavior in pottery design 55 in terms of (a) common hand \& finger movement patterns for 56 creating common geometric features, (b) user perception of in57 tent, and (c) engagement, utility, and ease of learning provided 58 by our approach.

\section{${ }_{59}$ 2. Related Work}

\section{2.1. Mid-air Gestures}

61 Gestures can be designed effectively for pointing, selec62 tion [13, 14], and navigation, since they define an unambigu63 ous mapping between actions and response. Such tasks are im${ }_{64}$ plemented using deictic gestures [15] and can usually be seg65 mented into discrete phases, with each phase triggering an event ${ }_{66}$ or a command [16]. Pointing in the direction of a virtual ob${ }_{67}$ ject creates the association between the user and the object. A 68 recent study [17] shows dwell-time to be an effective method 69 of pointing and selecting objects without hint to the users. In 70 manipulative tasks such as ours, a direct spatial mapping is re71 quired between the user's input and the virtual object $[18,15]$. 72 Particularly in our case, such an association would be in terms 73 of the proximity of the user's virtual hand to the shape being 74 deformed.

\section{2.2. Gestures for $3 D$ Modeling}

76 Let us consider a mid-air interaction scenario of selecting 77 and displacing a mesh vertex for deforming a 3D mesh. Since 78 the user's hands are interacting in the air, there is no physical or 79 natural mechanism for triggering events. Here, gestures could 80 serve two fundamental purposes. First, they help define a be81 ginning (e.g. reaching and clutching some region of interest) 82 and end (e.g. de-clutching the region after required deforma83 tion) of an interaction $[16,19]$. Secondly, they help define the 84 exact operation from a set of operations defined in the context 85 of the application. For example, the type of deformation could ${ }_{86}$ be selected by using different gestures (e.g. fist to pull, point to 87 push, open palm to flatten).

88 On these lines, most existing bare-hand interaction tech89 niques for 3D shape conceptualization, use gestures combined 90 with arm and full-body motions. Segen and Kumar [1] showed ${ }_{91}$ examples of computer-aided design (CAD) with their Gesture $92 V R$ system, using computer vision for general virtual reality ${ }_{93}$ (VR) applications. Wang et al. [2] presented 6D Hands to demon94 strate CAD using marker-less hand tracking. The modeling 95 of sweep surfaces using hand gestures and body motion was 96 demonstrated by Vinayak et al. [4, 5]. Han and Han [3] demon${ }_{97}$ strated an interesting surface-based approach with particular fo98 cus on audiovisual interfaces for creating 3D sound sculptures. 99 Holz and Wilson proposed Data miming [7] as an approach to100 wards descriptive shape modeling wherein voxel representation 101 of a user's hand motion is used to deduce the shape which the 102 user is describing. This approach uses hands without the ex103 plicit determination of gestures for recognizing the user's de104 scription of an existing shape.

\section{2.3. Hand Grasp}

106 Prehension is a common phenomenon in real-world inter107 actions. Jeannerod [20] notes two functional requirements of 108 finger grip during the action of grasping, (a) adaptation of the 109 grip to the size, shape, and use of the object to be grasped and 110 (b) the coordination between the relative timing of the finger 111 movements with hand transportation (i.e. whole hand move112 ments). Intended actions strongly influence motion planning of ${ }_{113}$ hand and finger movements [21]. This suggests that the intent 114 for deformation can be recognized before the user makes con115 tact with the surface being deformed. Grasp classification [22] 116 and patterns of usage and frequency [23] have been integral to 117 robotics research. Literature in virtual reality [24, 25] has stud118 ied and implemented grasping in the context of object manip119 ulation (pick-and-place). Kry et al. [26] implemented a novel 120 hardware system to emulate grasping for desktop VR applica121 tions such as digital sculpting. It is worth noting that the pri122 mary methodology for investigating grasp taxonomies is mostly 123 derived from the geometry of the hand in relation to a physical 124 object that is held or manipulated by the hand. What we aim to 125 do is to understand what is the minimal and sufficient character126 ization of the user's hand and finger movements, that could be 127 used for mid-air deformation. Our goal is not to explicitly de128 tect the hand grasp, but to design a deformation approach where 129 the grasp is automatically and implicitly taken into considera130 tion.

\section{${ }_{31}$ 3. Overview}

\section{3.1. Intent \& Controllability}

${ }_{133}$ The general term intent is literally defined as "the thing that 134 you plan to do or achieve : an aim or purpose". In our case, 135 intent (what one wants to achieve) can be described in terms 136 of the context of shape deformation (what operations one can ${ }_{137}$ perform on the shape). Based on Leyton's perceptual theory 138 of shapes [27], Delamé et al. [28] proposed a process gram139 mar for deformation by introducing structuring and posturing 140 operators. Here, structuring operators involve adding/removing 141 material to the shape, while posturing operators allow for modi142 fications such as bending or twisting some portion of the shape. ${ }_{143}$ Since our context is that of deformation, we define the intent in 144 terms of two basic operations: pulling and pushing. These are 145 analogous to structuring operators.

We see controllability as the quality of intent recognition 147 and disambiguation as perceived by the user. Specifically, in 148 our context, controllability is defined as a function of two fac149 tors: (a) the disparity between what a user intends for the shape 150 to be and what the shape actually becomes after the deforma151 tion and (b) the responsiveness of the deformation. The goal is 152 to minimize the disparity and optimize the responsiveness.

\section{3.2. Rationale for Pottery}

${ }_{154}$ We have two goals in this paper. First, we seek a con155 crete geometric method that takes a general representation of 156 the user's hand (PCL) and allows the user to deform 3D geom157 etry. Second, we want to investigate this geometric method in 

162 the hand. 185 tem.

158 light of intent and controllability. Thus, our focus here is not 159 to build a comprehensive and feature-rich 3D modeling sys160 tem. Instead, we intend to investigate spatial interactions for ${ }_{161} 3 \mathrm{D}$ shape deformation with an unprocessed representation of

${ }_{163}$ In a general shape deformation scenario, an arbitrary trian${ }_{164}$ gle mesh is the ideal and generic shape representation. How165 ever, a controlled study is prohibitively challenging in such a 166 case, for two reasons. First, the hand PCL data obtained from 167 a single depth sensor is partial and noisy. Second, dynamic and 168 complex finger motions add further complexity to the occlu169 sions and noise. Subsequently, designing interaction tasks for 170 a quantitative evaluation is difficult, particularly for users that 171 have no prior experience with mid-air interactions for free-form 172 3D modeling. Hence, it is essential to constrain the geometric 173 representation of the object being modified.

174 Our broader motivation in this work is to cater to the cre175 ative needs of individuals that are inclined towards 3D mod176 eling and design and but do not have the expertise require for 177 working with design tools. With this in view, we use pottery as 178 our application context for two reasons. First, it offers a well179 defined and intuitive relationship between the use of hands and 180 the shaping of pots to a user. This allows us to concretely con${ }_{181}$ struct a geometric relationship between the shape of the hand ${ }_{182}$ PCL and the corresponding user intent. Secondly, the simplic183 ity of the geometric representation and deformation lends itself 184 to quantitative measurement of the user's response to our sys-

\section{3.3. Approach}

${ }_{187}$ Given the context of pottery, our approach involved the fol188 lowing three stages:

Stage 1: Using hand as one-point manipulator, we implemented proximal-attraction, an interaction technique for clutching and de-clutching without hand gestures. Our technique (section 4) generalizes the notion of dwell-time in the context of mid-air shape deformation. We conducted a preliminary study to evaluate the feasibility and effectiveness of this technique.

Stage 2: We extended the proximal-attraction method to the whole shape of the hand (section 5) [11]. Here,the hand was represented as a collection of multiple points (i.e PCL) obtained via a depth sensor. Each point in the PCL deformed a small local region on the pot using the proximal-attraction approach. On the whole this amounted to a gradual and progressive convergence of the pot-profile to the shape of the user's hands. Through experimentation, we found that users had significant difficulty in creating convex (pulling) and flat (fairing) features on the pot. This method was also found to be agnostic to the user's grasp and hand movements.

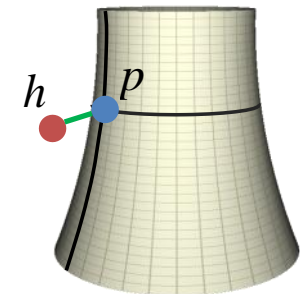

Compute closest point within distance threshold

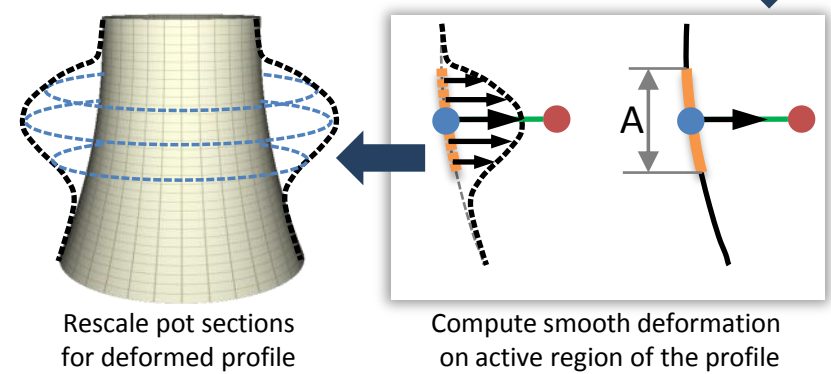

Figure 1: Algorithm for one-point pot deformation is illustrated for proximalattraction. The pot is gradually deformed by attracting the profile towards the hand (represented by a point). Subsequently, each section is re-scaled to obtain the deformed pot surface.

users' intent to push, pull or fair the surface of the pot depending on the hand grasp, finger movements, and motion of the 215 hand on the pot's surface. We conducted a final user evalua-

\section{3.4. Pot Representation \& Deformation}

${ }_{218}$ The deformation algorithm for the pot evolved through it219 erative implementation and evaluation. Here we describe the 220 basic geometric representation of a pot and the general compu221 tational setup of deforming the pot.

222 We represent a pot as a simple homogeneous generalized 223 cylinder. The surface of the pot is defined as a vertical stack 224 of circular sections. Each section is a polygonal approxima225 tion of a circle, i.e. a closed regular polygon. Note that a se${ }_{226}$ quenced list of pairs (radius, height) is the profile curve of the ${ }_{227}$ pot. The deformation of a pot is achieved by deforming the pro228 file curve, i.e by modifying the radii of each section. For a 3D ${ }_{229}$ pot, this essentially corresponds re-scaling each section by the 230 corresponding amount of deformation.

\section{${ }_{231}$ 4. Hand as a Point: Clutching by Proximal Attraction}

232 In the first stage, we developed a method wherein the hand 233 is represented as a single point manipulator, as is the case with 234 many gesture-based methods. The main goal was to allow users 235 to deform the surface the of pot without using hand gestures for 236 clutching and de-clutching the pot.

\section{Stage 3: Based on our experiments, we implemented our fi- 237 4.1. Technique}

nal technique for pot deformation using hand PCL (section 6). We used kernel-density estimation to characterize the contact between the hand and the pot. This allowed us to classify the
Let $h$ be the location of the hand in 3D space and $p$ be the 239 point on the pot that is closest to $h$. The main idea of proximal240 attraction is to deform the pot gradually by attracting $p$ towards 

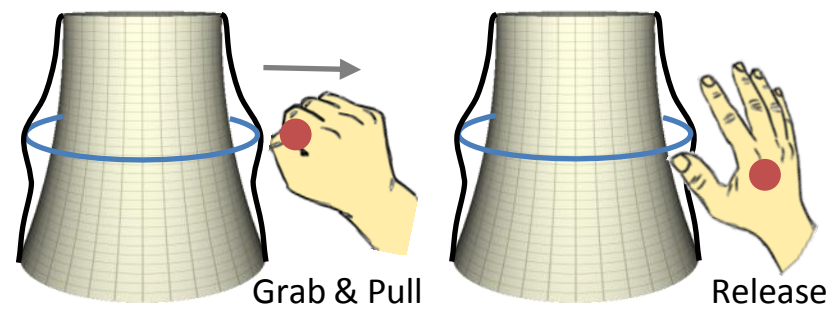

a. Gesture based deformation

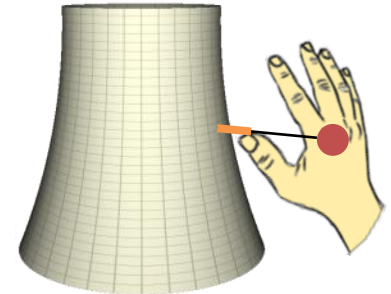

b. Proximal Attraction

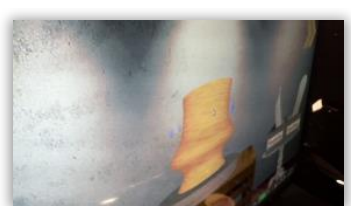

Screen

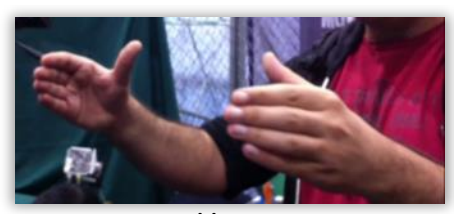

User
Figure 3: An example of common behavior is shown wherein users shaped their hands to express their intent for deformation.

269 the reception of a creative application such as pottery for a wide 270 variety of participants - particularly those without prior knowl271 edge of CAD tools. For this, we conducted a two-day field 272 study ${ }^{1}$ in an exhibition setting.

273 Apparatus. Our hardware setup consisted of a ThinkPad T530 274 laptop, a 60" display, and the Microsoft Kinect camera. The 275 Kinect camera was placed on a tripod below the display facing

Figure 2: Two strategies are shown for clutching and deforming a pot using hand as a single point. In the first approach (a) grab and release gestures. The second (b) is the proximal-attraction approach

${ }_{241} h$ in the horizontal plane. The condition of proximity is that 242 the distance between $h$ and $p$ should be less than a pre-defined 243 threshold (say $\varepsilon$ ). We implement the approach in the following 244 steps:

1. Given $h$ and $A$, compute $p$

2. if $(\|h-p\|<\varepsilon)$

(a) Set $\delta$ to horizontal distance between $h$ and $p$

(b) Set attraction at $p$ to $\alpha \delta$

(c) Compute smooth deformed profile using Laplacian smoothing $\left(\nabla^{2} \delta=0\right.$ for all points in $\left.A\right)$

\section{Rescale pot sections}

Here, $\alpha \in[0,1]$ is the rate of attraction where $\alpha=0$ implies 253 no attraction and $\alpha=1$ implies maximum attraction. Our idea 254 is inspired by exponential smoothing [29]. The main step was 255 to determine the right balance between the rate of attraction and 256 the distance threshold. The responsiveness of deformation is di257 rectly proportional to both, attraction rates and distance thresh258 old. From our pilot studies, we found $\alpha=0.3$ and $\varepsilon=0.05$ 259 to be the optimal values. Here, the distances are in the nor260 malized device coordinates. In our current implementation, we ${ }_{261}$ pre-defined the active region $A$ to be $50 \%$ of the total profile 262 length.

\section{4.2. Preliminary Evaluation}

264 Our main goal was to examine the feasibility and effec265 tiveness of the proximal-attraction approach for pot shaping 266 in terms of user performance and behavior. We also wanted 267 to determine the differences between our method and a typical 268 gesture-based approach. Additionally, we wanted to understand
276 a user standing at a distance of around 1.5 - 2.0 meters from 277 the display. Our pottery prototype was developed in $\mathrm{C}++$ and 278 openGL.

279 Implementation. We implemented two versions of our pottery 280 application, one using mid-air gestures and the other based on 281 the proximal-attraction approach. We first obtained the posi282 tion of the hand using the skeletal tracking algorithm provided 283 by the openNI API. Owing to the nature of the venue, the study 284 was not conducted in a controlled environment leading to dis285 turbances in skeletal tracking, posture recognition, and ambient 286 noise. Thus, appropriate measures were taken to isolate the user 287 from the audience.

288 The gesture-based prototype uses two simple hand postures, 289 grab and release, which correspond to closed and open palms 290 respectively (Figure 2(a)). We used the random forest algorithm 291 for posture recognition as detailed in [5]. The grab and release 292 postures allowed the user to clutch and de-clutch a certain re${ }_{293}$ gion of interest on the pot. The user could create concave and 294 convex profiles of the pot by grab-and-push and grab-and-pull 295 actions at the desired location of the pot surface in 3D space. In 296 the second prototype, we implemented our proximal-attraction 297 technique (Figure 2(b)). 299 (5-60 years) were invited to use our pottery prototype wherein, 300 the task for each participant was to create a pot as per the par301 ticipant's liking. Although we did not carry out a formal demo302 graphic survey, we found that the participants were from a va303 riety of backgrounds including non-technical users, engineers, 304 designers, artists, and professional potters. Our evaluation was 305 mainly informal and observational wherein we recorded videos 306 of sessions subject to the participant's permission and the time 307 taken to complete the creation of a pot. Due to the nature of our 308 venue, we constrained the maximum time for each participant 309 to about 8-10 minutes.

${ }^{1}$ MakerFaire, Bay Area (2013)
298 Participants \& Procedure. Participants within a wide age range 
Table 1: Behavioral observations in our preliminary evaluation

\begin{tabular}{|c|c|c|}
\hline Age & Value & Behavior \\
\hline $5-10$ & $\begin{array}{c}\text { Fun, } \\
\text { Play }\end{array}$ & $\begin{array}{c}\text { Excitement, } \\
\text { Random hand movements }\end{array}$ \\
\hline $11-15$ & $\begin{array}{c}\text { Entertainment, } \\
\text { Education }\end{array}$ & $\begin{array}{c}\text { Controlled movements, } \\
\text { Explored tool features }\end{array}$ \\
\hline $16-30$ & $\begin{array}{c}\text { Entertainment, } \\
\text { Art, Education }\end{array}$ & $\begin{array}{c}\text { Controlled movements, } \\
\text { Investigated pot behavior }\end{array}$ \\
\hline $30-60$ & $\begin{array}{c}\text { Entertainment, } \\
\text { Meditative }\end{array}$ & $\begin{array}{c}\text { Controlled movements, } \\
\text { Expected real-world like response }\end{array}$ \\
\hline
\end{tabular}

310 A total of 360 participants responded to our invitation and 311 used our prototype to create pots. In the first session (day 1), 312180 participants used the prototype implemented using the grab 313 and release gestures. In the second session (day 2), 180 partic314 ipants used the proximal-attraction technique for pot deforma315 tion. There were participants that were either completely unable 316 to create any meaningful shape of the pot or did not find the re317 sulting shape as the intended one. These attempts we removed 318 from our database leaving us with the recorded times for 113 319 participants per session (i.e. 226 participants in total).

\section{4.3. Results}

321 We categorized the perceived value and user behavior dur322 ing the use of the pottery applications on the basis of age. Young ${ }_{323}$ participants (5-10 years) were mostly interested in simply play324 ing around with the application and usually applied arbitrary 325 hand movements during the deformation of the pot's profile. ${ }_{326}$ Participants in the age range of 11-15 years provided more con327 trolled movements of the hands during pot shaping with slower 328 and more careful hand movements and accurate hand gestures. ${ }_{329}$ They also adopted a more exploratory approach towards the ap${ }_{330}$ plications in that they were primarily interested in the various ${ }_{331}$ software features rather than the realism in the pot's deforma332 tion.

3зз However, in case of participants above the age of 15 , we 334 observed that they instinctively shaped their hands according to ${ }_{335}$ geometry of the pot on the screen. Specifically, users within 33616 and 30 years of age were mainly interested in investigating 337 how the gesture and motion of the hand was related to the de338 formation of the pot. They would frequently expect the pot to ззя deform according to how they shaped and moved their hands 340 on the pot's surface. This strongly suggested that the internal 341 learning of physical interactions, combined with some prior ex342 pectation of the pot's response, increased with the participants' 343 age. In case of the gesture-based approach, this was also a cause 344 for intermittent gesture misclassification, resulting in user frus345 tration. Despite their simplicity, the grab and release gestures 346 were tedious to use while using virtual tools. This was mainly 347 the case with participants who were completely new to inter348 faces developed for RGBD cameras.

349 On the other hand, users found the proximal-attraction ap350 proach easier to learn and use. The participants could immedi351 ately start deforming the pot, and at the same time they could 352 shape their hands as they saw fit. A common mental model that
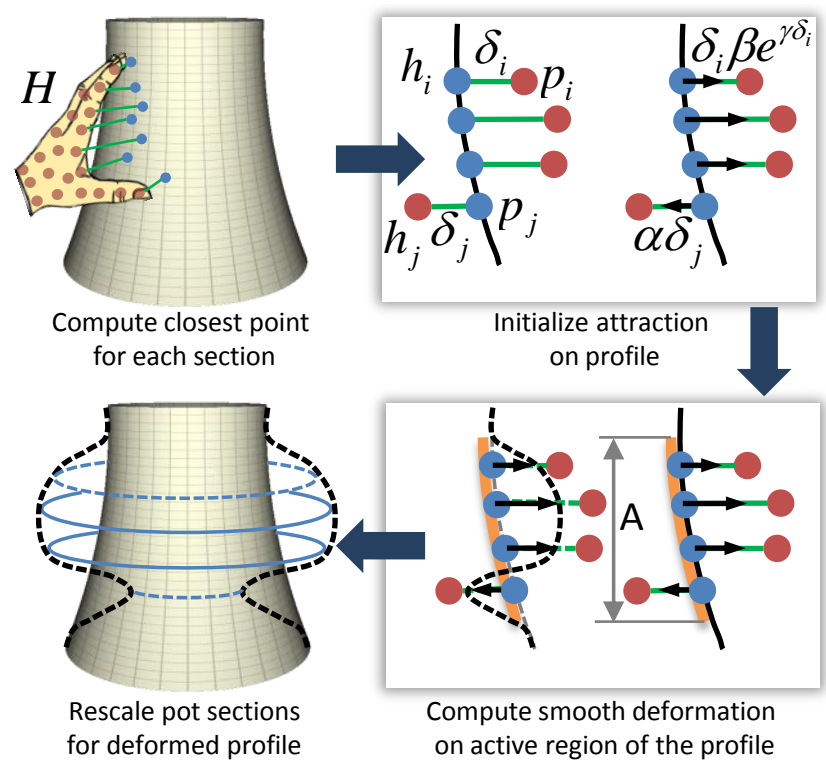

Figure 4: Algorithm for pot deformation is illustrated for proximal-attraction. The profile is deformed based on the proximity of the points on a given hand PCL. Subsequently, each section is re-scaled to obtain the deformed pot surface.

353 the users seemed to create was that of a surface which "sticks" 354 to their hands upon coming close. Thus, the users were in355 variably slower while approaching the pot (so as to reach the 356 right location) and retreated faster when they wanted to release 357 contact with the pot. For some users, fast retreat also caused 358 accidental deformation leading to frustration.

\section{4.4. Takeaways}

The two main insights we gained were: (a) the intent for de361 formation directly translates to how users shape their hand and 362 (b) the rate of attraction for pulling and pushing must be deter363 mined separately so as to make them consistent. We found that 364 full-body interactions caused significant fatigue and difficulty 365 in controlling deformation. Thus, our subsequent stages, we 366 implemented interactions at close range wherein a user could 367 perform pottery sitting in front of a desktop or a laptop com368 puter.

\section{5. Hand as a PCL: Shaping by Proximal Attraction}

370 Our main objective in this stage was to adapt the proximal371 attraction method that could use the shape of the whole hand to 372 deform the pot. Thus, we used a representation of the hand as 373 a collection of multiple points (i.e PCL) obtained via a depth 374 sensor.

\section{5.1. Technique}

376 Consider the hand $H$ as a set of points $\left\{h_{i}\right\}$ in 3D space. Each 377 point in the PCL deforms a small local region on the pot using 378 the proximal-attraction approach. On the whole this amounts to 379 a gradual and progressive convergence of the pot-profile to the 380 shape of the user's hands (Figure 4). 


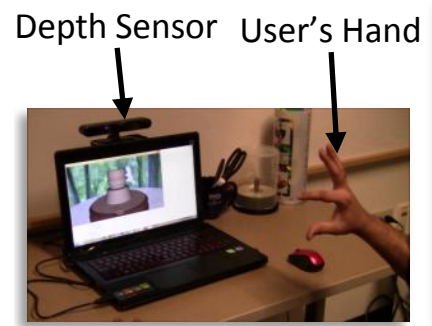

a. Apparatus

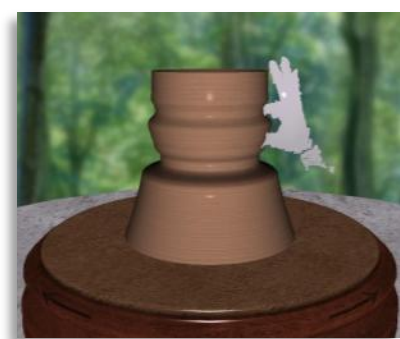

b. Interface
Figure 5: The apparatus (a) consists of the user, a computer and a depth camera. The user sees a PCL of their hand deforming a rotating pot (b).

${ }_{381}$ Pushing vs. Pulling. A push is characterized by an inward dis382 placement $(\delta<0)$. This is the simplest case wherein a user 383 would typically approach the pot and subsequently recede away 384 once the desired deformation has occurred. A pull is character385 ized by an outward displacement $(\delta>0)$. This is a non-trivial 386 intent to recognize since a user would invariably approach the 387 surface first and then recede to pull. The overall motion of 388 the hand is similar to that of a push. In order to distinguish 389 pulling and pushing, we used two different rates of attraction. 390 For pulling, we defined the attraction rate as a smooth function 391 of the distance between the hand point and pot. The function 392 is given by $\beta e^{\gamma \delta_{i}}$. For pushing, we defined the rate of attrac393 tion as $\alpha$. This essentially allows the user to first approach the 394 pot without deforming it during the process of approach. The 395 algorithm is as follows:
409 Initialization Time. In order to avoid accidental or unintended 410 deformation of the pot, we implemented an that allows for the 411 pot to deform only when contact with the pot is maintained for a 412 sufficient amount of time. We achieved this in two steps. First, 413 we reset $\alpha$ and $\beta$ to 0 at every new contact that the hand made 414 with the pot. Subsequently, we linearly increase them to their Compute unique $h_{i}$ such that $\left\|h_{i}-p_{i}\right\|<\varepsilon$ is minimum.

Set $\delta_{i}$ to horizontal distance between $h_{i}$ and $p_{i}$

2. Set $\delta_{r}$ to $\delta_{\max }-\delta_{\min }$

3. Set $\gamma$ to $\frac{0.1}{\delta_{r}}$

4. For each $i$ on profile

if $\left(\delta_{i}<0\right)$ : Set attraction at $p_{i}$ to $\alpha \delta_{i}$ else: Set attraction at $p_{i}$ to $\beta e^{\gamma \delta_{i}} \delta_{i}$

\section{Compute Active region $A$}

6. Smooth deformation $\left(\nabla^{2} \delta=0\right.$ for all points in $A$ )

7. Compute deformed profile

\section{Rescale pot sections}

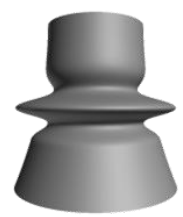

a

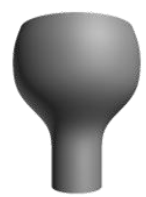

e

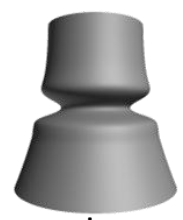

b

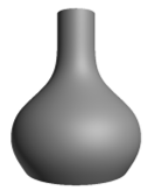

f

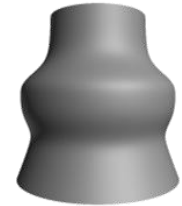

C

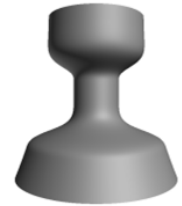

g

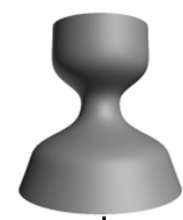

d

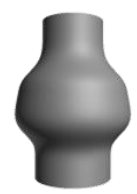

$\mathrm{h}$
Figure 6: Eight pre-defined pots were shown to participants in the quiz. These are: $(a, b)$ thin convex and thin concave, $(c, d)$ fat convex and concave, $(e, f)$ round and flat, and $(\mathrm{g}, \mathrm{h})$ flat at center and ends. (from Vinayak et el. [11])

415 maximum values within a stipulated amount of time $T$. We call ${ }_{416}$ this the initialization time. Intuitively, $T$ is the time taken by 417 the pot to gradually initiate the response to the user's hand after 418 a contact is made.

\section{5.2. Experiment}

We conducted a lab experiment to evaluate the proximal421 attraction approach. The results of this experiment led us to 422 develop the final approach in this work. In the paragraphs be423 low, we will describe selective details of our prior work for the 424 sake of completeness. For a comprehensive analysis of this ex425 periment, the reader can refer to our prior published work [11].

426 Apparatus. Our setup consisted of a Lenovo ideaPad Y500 lap-

${ }_{427}$ top computer with an intel i7 processor and 8GB RAM, running ${ }_{428}$ 64-bit Windows 8 operating system with a NVIDIA GeForce ${ }_{429}$ GT 750M graphics card, and the SoftKinetic DS325 depth sen430 sor (Figure 5(a)). SoftKinetic DS325 is a close range $(0.1 \mathrm{~m}$ ${ }_{431} 1.5 \mathrm{~m}$ ) time-of-flight depth sensor that provides a live video stream 432 of the color and depth image of the scene. Every pixel on a ${ }_{433}$ given depth image can be converted to a 3D point using the ${ }_{434}$ camera parameters.

435 Implementation \& Interface. After segmenting the hand from 436 the scene, we use the SoftKinetic iisu API for tracking the hand ${ }_{437}$ PCL. However, the tracking method provided in this API does 438 not work with hand-held objects - a feature that we required 439 in order to allow users to utilize physical objects for deforma440 tion. Thus, we used a pre-defined a volumetric workspace as 441 the active region in front of the computer screen. Our inter442 face comprises of a 3D scene with a rotating pottery wheel on 443 natural outdoor background (Figure 5(b)). The user sees the ${ }_{444}$ potter's wheel and the PCL of their hands, or the tools held in 445 their hands. We designed this interface based on the guidelines 446 provided by Stuerzlinger and Wingrave [30]. Finally, we pro447 vided keyboard shortcuts to the allow the participants to undo 448 and redo a particular deformation at any time. Additionally, 449 we also made provisions for the participants to reset the current 450 shape to the blank pot. 
${ }_{451}$ Participants. The participants of this evaluation comprised of 45215 (13 male, 2 female) science and engineering graduate stu453 dents within the age range of $20-27$ years. Out of the 15 ${ }_{454}$ participants, 5 participants self-reported familiarity with mid455 air gestures and full body interactions through games (Kinect, ${ }_{456} \mathrm{Wii}$ ). Due to engineering background, most participants (12 of 457 15) reported familiarity with 3D modeling and computer-aided 458 design. Incidentally, we also had 3 participants who had prior 459 experience with physical ceramics and pottery.

460 Procedure. The total time taken during the experiment varied 461 between 45 and 90 minutes. We began the study with a demo462 graphic surface where we recorded participants' background re${ }_{463}$ garding their familiarity with depth cameras, full-body games, 464 and pottery. Subsequently, we provided a verbal description 465 of the setup, the purpose of the study, and the features of the 466 pottery application. This was followed by a practical demon${ }_{467}$ stration of the pottery application by the test administrator. The 468 participants were then asked to perform the following tasks:

\section{5.3. Results}

499 The following paragraphs briefly summarize the observa500 tions that we have detailed in our prior work [11].

501 Reaching, Grasping, \& Deformation Strategies. Each user had 502 a different perception of the process necessary to achieve the 503 profile of a given target shape. Most users attempted the quiz
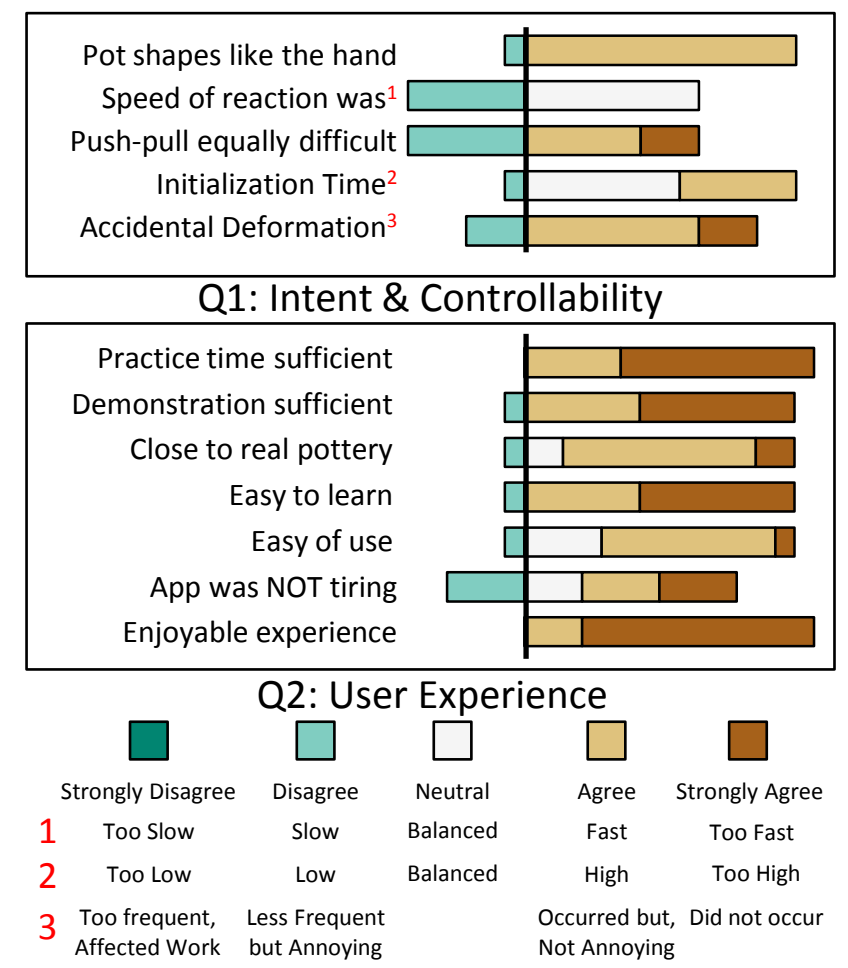

Figure 7: User response to are shown for proximal-attraction. The main issue in terms of controllability (a) was the slow response and difficulty of pushing in comparison to pulling. (from Vinayak et el. [11])

504 problems in multiple trials, wherein they would refine their strat505 egy to deform the profile in every trial. However, we observed 506 that these strategies of reaching, grasping, and deforming the 507 profile converged to patterns common across users (Figure 8). 508 Typically, users would first estimate the size and shape of the 509 grasp according to the geometric feature of the profile and then 510 move the whole hand in the intended grasp to deform the pro511 file [21]. The most common usage pattern observed across 512 users was the recursive smoothing and refining of the pot after ${ }_{513}$ deforming the profile reasonably close to the target shape. This 514 was typically done by moving the hand vertically along the sur515 face of the pot (Figure 8). This was the cause of frustration for 516 two reasons. First, the accidental contact of the hand with the 517 pot's surface resulted in unintended deformations. Second, the 518 proximal attractions did not allow for an explicit way to smooth 519 or straighten a region of the pot. Despite being reminded of the 520 undo, redo, and reset functionalities, most users preferred us521 ing their hands for reversing an accidental deformation. For the 522 thin-convex profile, most users first created a convex feature in 523 the center followed by pushing the top and bottom portions in524 ward. For concave features, users first pulled the top and the 525 bottom portions of the pot and subsequently pushed the cen526 tral region of the pot (Figures 9(a)). This was an interesting 527 common pattern since we had assumed that users will create 528 concave features in a single inward action. This was also the 529 case with flat-round features (Figures 9(b)) wherein many users 530 first pulled out the round feature followed by straightening the 


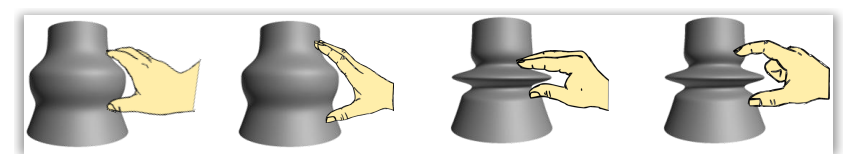

a. Pulling

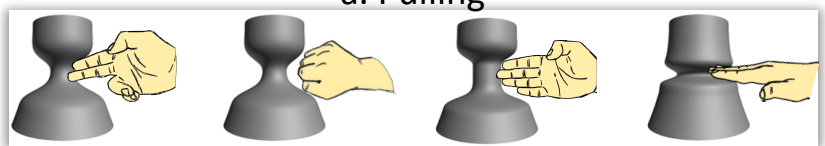

b. Pushing

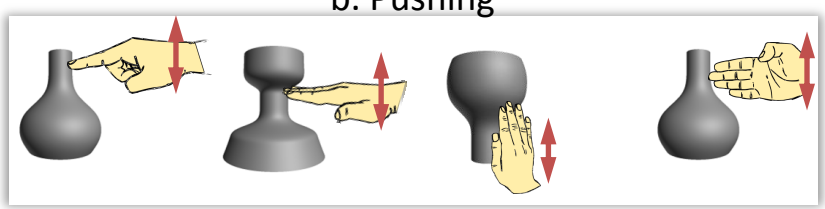

c. Smoothing

Figure 8: Common user patterns are shown in terms of grasp and motion performed by users for each target shape (in decreasing order of occurrence along columns). The hand images represent the grasp and the arrows (red) show the motion of the hand. The most successful strategies are indicated by blue boxes for each target shape.

531 flat regions of the pot. The pointing posture of the hand was 532 commonly observed during the creation of thin concave fea533 tures. However, in subsequent trials, most users resorted to us534 ing an open palm. This was because the pointing pose limited 535 the depth to which the users could push the surface inwards, 536 owing to the interference of the fingers other than the index 537 finger. The cupping of the hands in conjunction with vertical 538 movement of the hands was a common approach for round fea539 tures.

540 The use of two hands was particularly prevalent for round${ }_{541}$ flat combinations. Due to arm fatigue, some users also changed 542 from their dominant hand to the non-dominant hand. This was a 543 cause for frustration due to the limited volume of the workspace 544 and unintended deformations caused by the asynchronous mo545 tions of two hands. Most users commonly approached the pot 546 from the sides. The reason, as stated by a user, was: " $m y$ own 547 hand blocks the view of the pot". Difficulty in depth percep548 tion caused many users to inadvertently reach behind the pot's 549 surface. This caused further unintended deformations when the 550 user did not expect one, or the lack of response when it was 551 expected.

${ }_{552}$ Intent \& Controllability. In general, users agreed that the shape 553 of the profile behaved in correspondence to shape of the hands 554 (Figure 7(a)). However, only $50 \%$ of the users agreed that the 555 response speed of the deformation was balanced. There was a 556 common agreement on the initialization time and robustness to 557 accidental deformation. There were two common and expected 558 difficulties that the users faced. These were: (a) pulling specific 559 regions of the pot and (b) creating straight and flat features on 560 the top portion of the pot. As a user stated: "Pushing seems 561 easier than pulling. Part of the reason I suspect is the visual ${ }_{562}$ feedback. It is easier to determine if my hand starts to touch 563 the pot, while it's not as easy to determine if my hand is still ${ }_{564}$ attached with the pottery or leaving it.". This indicated that
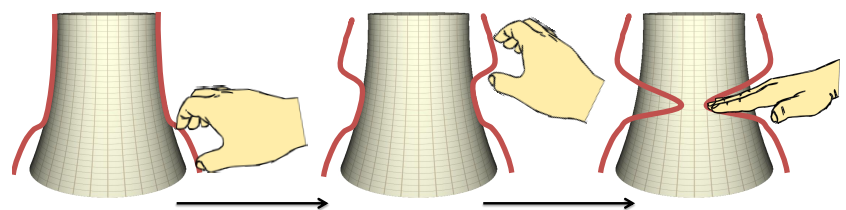

a. Creating a thin concave feature

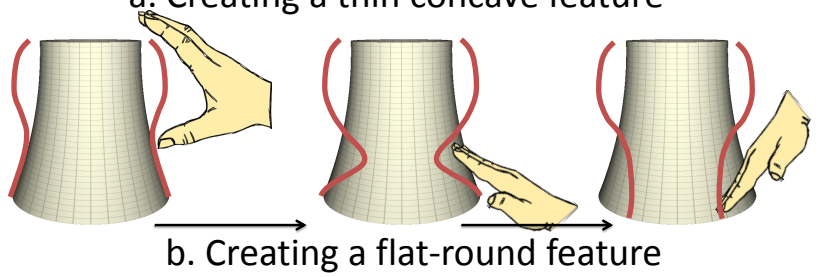

Figure 9: Two examples are shown of common deformation strategies are shown through which users created (a) thin concave and (b) flat-round features. (from Vinayak et el. [11])

565 perceiving the depth difference between the hand and the pot 566 was difficult for the users.

\section{5.4. Takeaways}

568 There were two main issues with the proximal-attraction ap569 proach. First, pulling was clearly more difficult since the rate 570 of attraction was designed to be lower than that of pushing. 571 Secondly, the users clearly distinguished between several op572 erations of fairing, straightening, carving, pulling and pushing. 573 However, the proximal-attraction approach, was not designed 574 to explicitly identify or classify the type of operation the user 575 intended to perform. Our main goal in our third and final stage 576 was to resolve these two issues. Our first step was to identify 577 the main characteristics of users' preferences towards grasp578 ing to pull and motion patterns for smoothing the pot. Subse579 quently, the aim was to design a geometric approach that could 580 recognize these identified characteristics and broadly classify 581 the intended actions from the hand PCL.

\section{${ }_{82}$ 6. Hand as a PCL: Grasp + Motion}

Our observations strongly indicated that users distinguished 584 their intent in three broad categories: pulling, pushing, and 585 smoothing. In our final stage, we implemented a grasp and mo586 tion based approach to identify these three classes of intent.

\section{6.1. Technique}

The basic idea of the grasp+motion approach is to summarize the grasp of the hand in relation to the surface of the pot and subsequently classify the user's action (Figure 10). We achieve this by using kernel-density estimation of the point cloud on the axis of the pot. In our context, this kernel-density estimate (KDE) is essentially a smoothed histogram of the distribution of the hand's PCL on the pot's. We use the exponential function to determine the KDE. For a given section $i$, the $\mathrm{KDE}$ is given by:

$$
\phi_{i, j}=\sum_{j=1}^{j=|H|} e^{a\left\|\delta_{i, j}^{2}\right\|}
$$




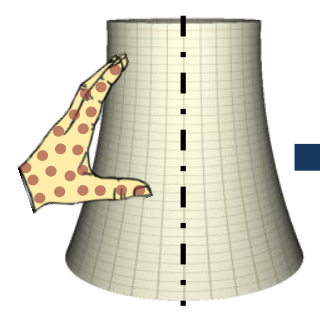

Input: Hand PCL and Pot

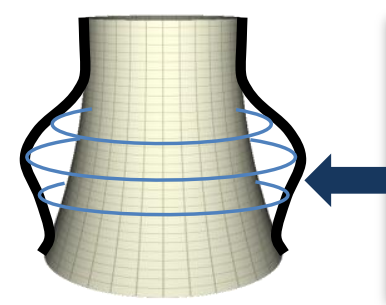

Rescale pot sections for deformed profile

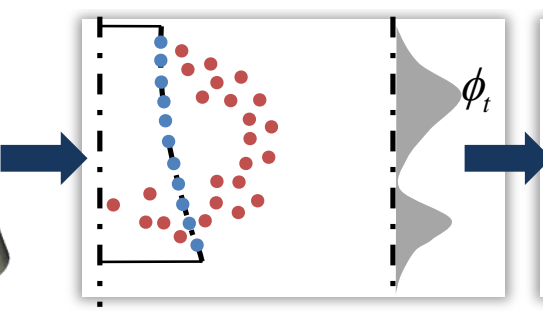

Compute KDE on Pot's Axis

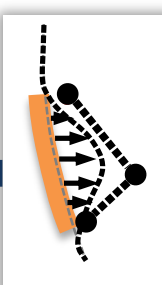

Compute Rate of Attraction using

Pulling Function on Active region
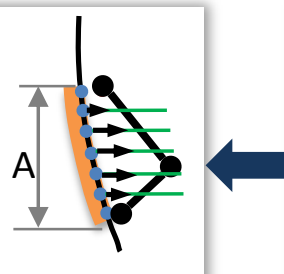

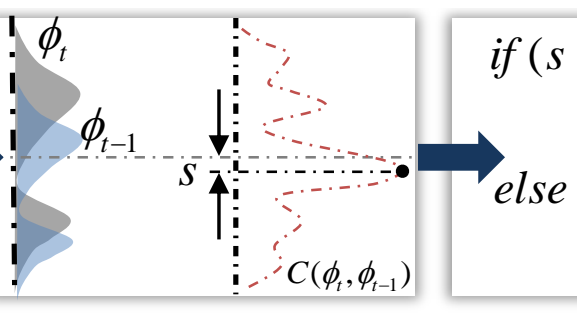

Cross-correlation with previous KDE

Figure 10: Algorithm for grasp+motion technique is illustrated. The main steps involve computation of axial KDE for hand PCL, detection of intent for smoothing, differentiation between pulling and pushing, and deformation of the pot. In this example, we show the details of the pulling deformation (row 2).

588

There were three main observations (Figure 8) that helped 589 us use the KDE to classify the user's intent. First, users moved 590 their hands in a fixed pose along the surface of the pot to ex${ }_{591}$ press their intent for smoothing. This corresponds to detect592 ing the vertical shift of the KDE. We used normalized cross${ }_{593}$ correlation [31] between the two consecutive KDE signals to 594 determine the shift. Secondly, for pulling the pot, we observed 595 that users used specific grasps. In this case, we note that the ${ }_{596} \mathrm{KDE}$ has two maxima and one minima (Figure 11). Here, each 597 maxima corresponds to the fingers making contact with the pot 598 and the minima corresponds to the center of the grasp. This 599 essentially allows us to track a basic skeletal representation of 600 the hand. We then define the attraction rate using a based on 601 the angle of grasp $(\phi)$ (Figure 12). Finally, all actions that do 602 not correspond to either smoothing or pulling, are assigned as ${ }_{603}$ pushing. For pushing, we use the proximal-attraction approach 604 for deformation. The steps of the algorithm are:

Compute extrema

Detect skeleton

Compute $\theta$

if $(\#$ maxima $=2 \& \theta<2 \pi)$ : Apply pulling in $A$ else: Apply proximal-attraction in $A$
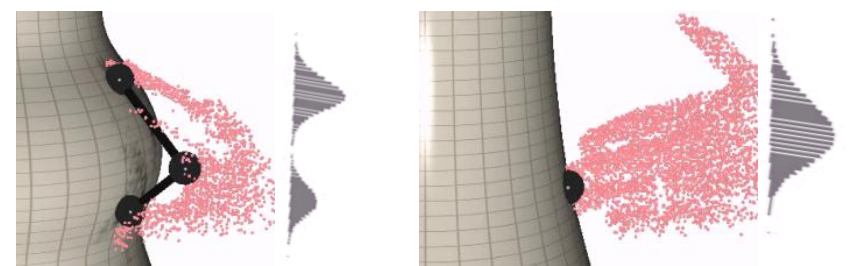

Figure 11: KDE functions are shown for a pulling (left) and pushing (right) intents.

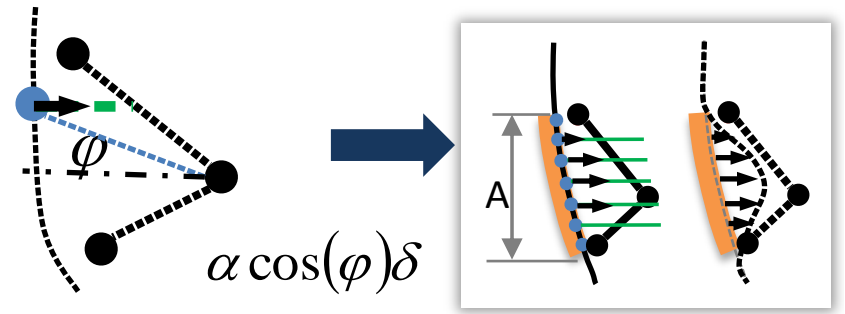

Figure 12: Computation of attraction rate using the angle of grasp.

7. Smooth deformation $\left(\nabla^{2} \delta=0\right.$ for all points in $\left.A\right)$

8. Compute deformed profile

9. Rescale pot sections

\section{6.2. Experiment}

${ }_{620}$ We used identical apparatus and interface to evaluate our ${ }_{621}$ final stage. Additionally, we made two important modifications 622 to the interface. First, we added a shadow of the hand on the ${ }_{623}$ surface of the pot. The goal was to enable users to estimate their 


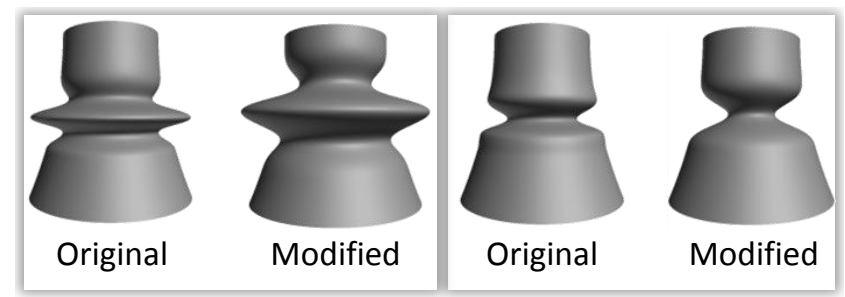

a. Thin Convex

b. Thin Concave

Figure 13: The thin convex and concave features were modified according to the capability provided by the grasp+motion technique.

624 proximity to the surface. Secondly, we clamped the hand PCL 625 So as not to allow points on the hand to reach behind the surface 6 626 of the pot.

${ }_{627}$ Participants. We recruited 15 (11 male, 4 female) participants 628 within the age range of $19-30$ years. None of these partici629 pants had prior knowledge of mid-air interactions or had par630 ticipated in any of our previous studies with pottery interface. ${ }_{631}$ All participants were from science and engineering background 632 wherein 10 participants had familiarity with mid-air gestures 633 and full body interactions, and 11 participants reported familiar${ }_{634}$ ity with 3D modeling and computer-aided design. 5 participants 635 reported that they had practical familiarity with real ceramics 636 via informal workshop sessions but did not pursue pottery as a 637 regular activity or professional practice.

${ }_{638}$ Procedure. Our overall experimental procedure was identical 639 to the one that we used for evaluating the proximal-attraction 640 approach (Section 5.2, Procedure). However, we made three ${ }_{641}$ modifications to the evaluation procedure as listed below:

1. One of the main goals of our work was to enable users to invoke their tacit knowledge of deforming physical objects. To this end, we designed the grasp+motion approach such that it is geometrically-driven and can potentially be used even for user inputs that used other physical objects as tools in addition to the use of hands. In order to verify the generality of our approach with respect to user input, we added another composition task (T3) wherein participants were given a duration of five minutes to create pots using a set of physical artifacts as tools. Our "tools" comprised of day-to-day objects (e.g. white-board marker, pair of scissors, ruler) and also some special objects such a Shapescapes ${ }^{\text {TM2 }}$.

2. In order to understand user experience with physical objects tools, we also added questions to the questionnaire Q2 regarding the utility, ease of use, and preference of tools over hands.

3. We modified the target shapes for the thin convex and concave features (Figure 13). The rationale behind this

${ }^{2}$ www.shapescapes.com

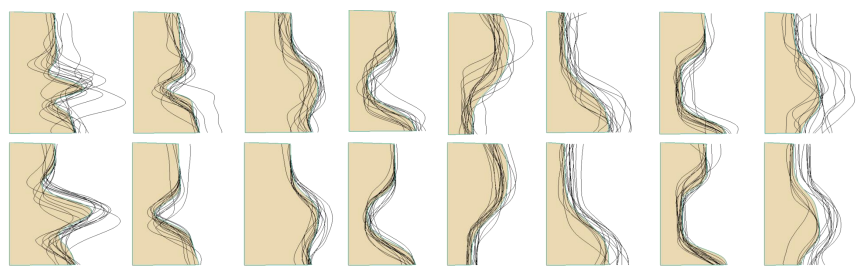

Figure 14: User created pot profiles (black curves) are shown relative to the target shapes (light brown cross sections). The top and bottom rows shows the results for proximal-attraction and grasp+motion approaches respectively. Visual inspection evidently shows improvements in the creation of flat, round and smooth features. More significant improvements were observed in the creation of fat convex features in comparison to proximal-attraction. 666 the completion time and the profiles of the pots shaped by the 667 users. Even though we designed T1 towards statistical analysis, 668 we observed that each user perceived the target shapes differ669 ently and consequently the measured data did not provide suf670 ficient insights regarding the strengths and weaknesses of our 671 approach. With this in view, we present a visual comparison of 672 the numerical data recorded during the evaluation of proximal673 attraction and grasp+motion techniques.

674 6.3. Results

675 User Performance (T1). Visual similarity with respect to the 676 target shapes evidently increased in comparison to the proximal677 attraction approach (Figure 14). This was primarily due to the 678 explicit smoothing. Overall, the completion time (Figure 15(a)) 679 was reduced as expected. Surprisingly, the maximum comple680 tion time across all users and all target shapes was recorded for 681 the thin-concave feature (14.4 minutes) followed by the thin682 convex feature (13.2 minutes). The mean completion time was 683 highest for the thin-convex feature (3.4 minutes) followed by 684 the central-flat feature (3.3 minutes). The main aspect that we 685 sought from T1 was the quality of the final outcome across par686 ticipants for a given quiz problem. We used curvature cross687 correlation $(C C C$ ) as a measure of the quality of user created 688 profiles (see [11] for details). As expected, the smoothness of 689 the results was notably superior in comparison to the proximal690 attraction (Figure 15(b)). We also recorded the number of tri691 als per user per target shape (Figure 15(c)). The global maxi692 mum number of trials were 7 and 5 for proximal-attraction and 693 grasp+motion techniques respectively. In case of grasp+motion, 694 most users required only one trial for fat-convex, central-flat, 695 and top-bottom-flat features. On the other hand, thin-concave 696 and thin-convex features required more iterations.

697 Each user perceived and approached a given target shape in 698 different ways. Consequently, there was no evident correlation 699 between the time taken by each user and the quality $(C C C)$ of 700 the final pot created by the user for any of the target shape. To 


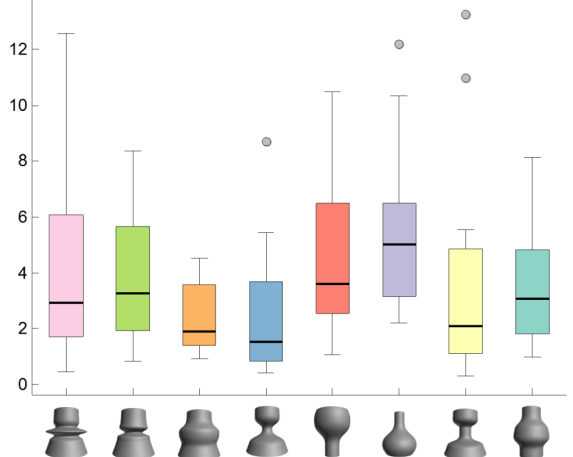

15
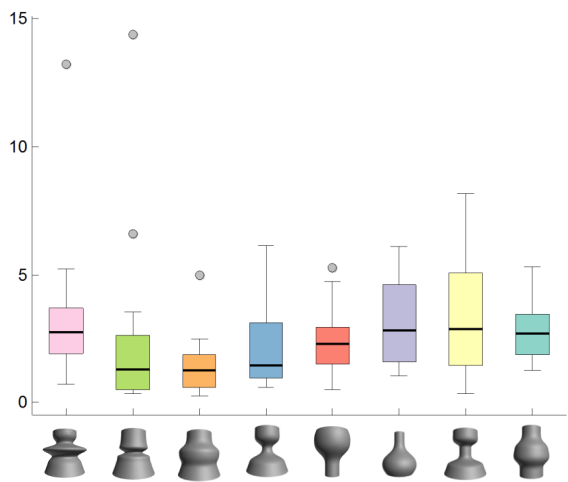

(a) Time (mins)
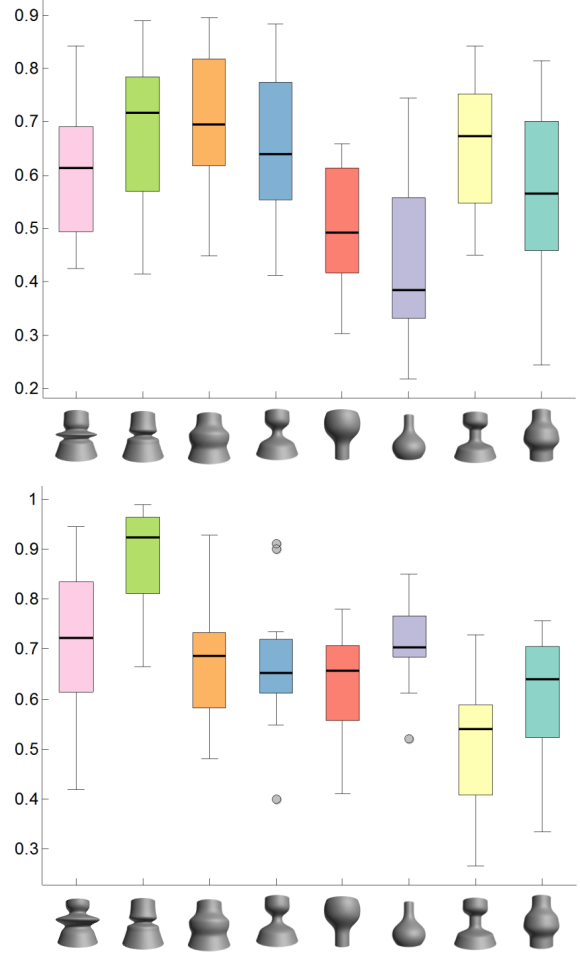

(b) Curvature Cross-correlation (0-1) $\square 1 \square 2 \square 3 \square 4 \square 5 \square 6 \square 7$

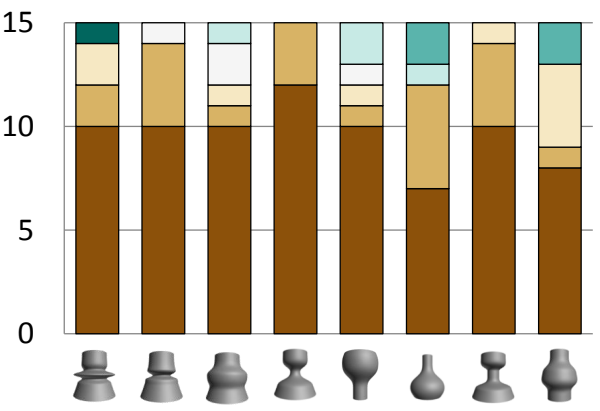

$\square 1 \square 2 \square 3 \square 4 \square 5$

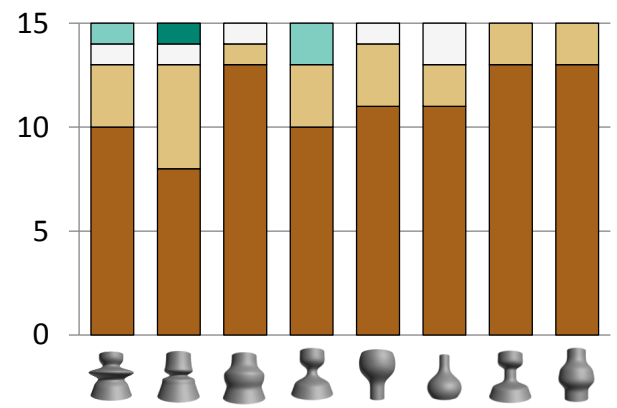

(c) Trials per user

Figure 15: A comparison between proximal-attraction (top row) and grasp+motion (bottom row) is shown in terms of (a) the time taken by users to shape a target profile, (b) the quality of users' responses in terms of curvature cross-correlation of profiles, and (c) the distribution of users with respect to the number of trials per target profile.
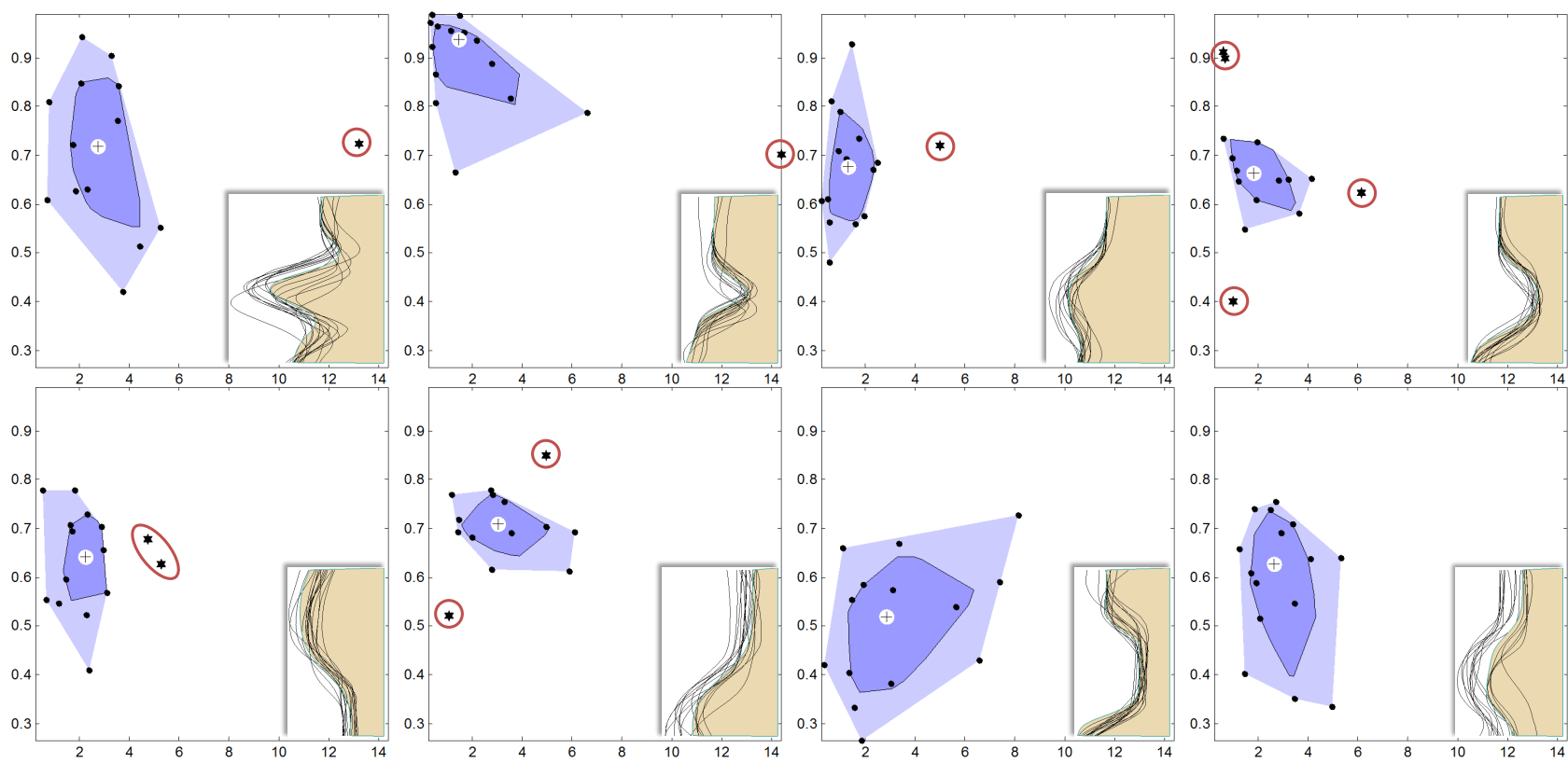

Figure 16: User performance is shown for the each quiz problem as a bag-plot. The x-axis is time in the range [0,14] minutes and the $y$-axis is the curvature cross-correlation in the range $[0,1]$. The dark and light blue regions show the bag and fence regions, respectively. The white circle is the Tukey depth median and the points marked with red circles are the outliers. The insets show the actual pot profiles (black lines) created by the users in comparison to the target shapes (beige region) of the Quiz. The coordinates of the depth median (C) and the spread (Sp) are provided for each target shape. 

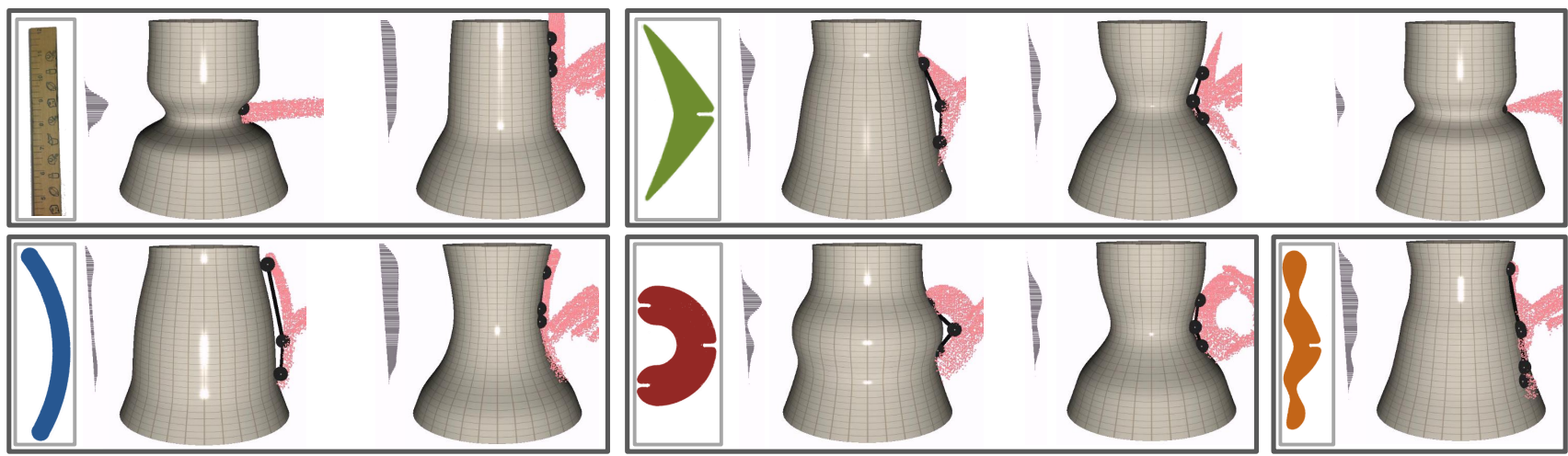

Figure 17: The characterization of tool geometry is visualized for five different physical objects. The objects were chosen to represent concave, convex, flat, and round contacts for deformation.

701 account for this, we represent the user performance as a bivari702 ate dataset given by the ordered pair of the response quality and 703 completion time. We visualize performance as a bag-plot [32] 704 (Figure 16). Here, the spread of the data (i.e. variations in user 705 responses) is given by the area of the bag. Users clearly per706 formed best for thin-concave targets with Tukey median value 707 of $(0.94,1.46)$. Performance was most consistent for the fat708 concave feature (Figure 16(d)). Users also performed consis709 tently for round-and-flat features (Figures 16(e) and (f)). Vari710 ations were significant for central flat feature (Figure 16(g)). ${ }_{711}$ Further, the pot-profile quality was very low for the central-flat 712 and top-bottom-flat features (Figures $16(\mathrm{~g})$ and $(\mathrm{h})$ ). This was 713 mainly because users typically spent considerable time pulling 714 and smoothing the top and bottom regions after performing an 715 initial push. Consequently, the median completion times were 716 also higher for the round-flat and central-flat features (Figure 71716 (f) and (g) respectively).

${ }_{718}$ Hand Usage (T1). The general user behavior in terms of reach719 ing the pot was similar to the proximal-attraction approach. 720 Both the algorithm and its description was different in this case. ${ }_{721}$ The users were explicitly made aware of pushing, pulling and 722 smoothing as three distinct operations. This obviously led to 723 variation in user behavior as compared to proximal-attraction.

724 Hand Usage (T2). On average, users created 5 pots (max: 12, 725 min: 2) within 5.80 minutes (std: $0.66 \mathrm{~min}$ ). We made two in726 teresting observations in T2. First, we found that users were 727 able to repeat the process of getting from an initial shape to 728 the same final shape across multiple trials. Similarly the users 729 could also deform a current shape back to some previous shape, 730 akin to the undo operation, but with the hands. In fact, most par731 ticipants preferred using their hands to undo a pot deformation 732 instead of the keyboard-shortcut. One user stated: "I thought it 733 was easier to learn the software when I was trying to make my 734 own pot not a model one". This was expected because of the 735 learning and practice that the users had during the quiz (T1). 736 However, during T1, users mentioned that their attention was ${ }_{737}$ divided due to the need to intermittently look at the target shape 738 during the shaping process. Thus, they generally perceived T1 739 to be more demanding than $\mathbf{T} 2$.

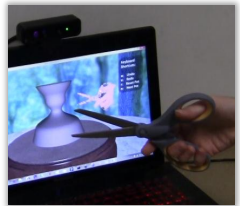

a.

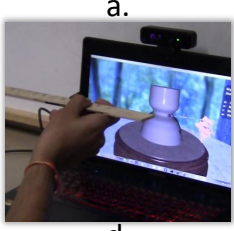

d.

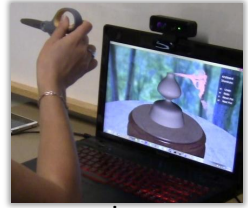

b.

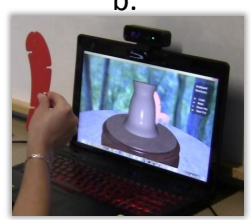

e.

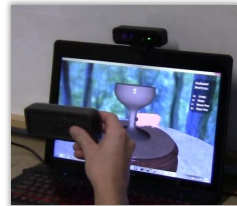

c.

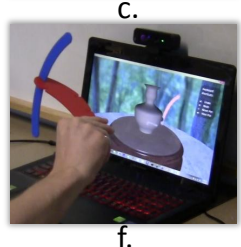

Figure 18: Examples of tool usage are shown.

740 We made two observations that were not evident in the ear741 lier stages. First, we found that the ability to repeat the process 742 of getting from an initial shape to the same final shape. Simi${ }_{743}$ larly, the ability to get to some previous state from the current 744 state was increased substantially. We observed that most of the 745 participants were successfully able to use their hands to undo a 746 pot deformation instead of the keyboard-shortcut.

${ }_{747}$ Geometric Characterization of Tools. The choice of everyday 748 objects and ShapeScapes ${ }^{\mathrm{TM}}$ was mainly helpful in providing a 749 reasonable variety of geometric profiles for pot deformation. 750 However, in order to better understand how users would use 751 these objects, we wanted to pre-determine how the intent of 752 pulling and pushing translates to the use of physical objects. 753 Thus, we conducted a set of experiments (Figure 17) to ver754 ify if the users could in fact extend their understanding of the 755 grasp+motion approach and apply it to the use of physical tools. ${ }_{756}$ Our experiments showed that the geometry of the tool can in757 deed be interpreted in terms of the nature of the KDE of the 758 tool's PCL and the grasping angle of the skeleton computed 759 from the KDE. Below, we summarize how this observation came 760 into play during the usage of tools by our participants.

761 Tool Usage (T3). Users showed immediate enthusiasm during 762 the use of tools. Almost all users first inspected the objects 

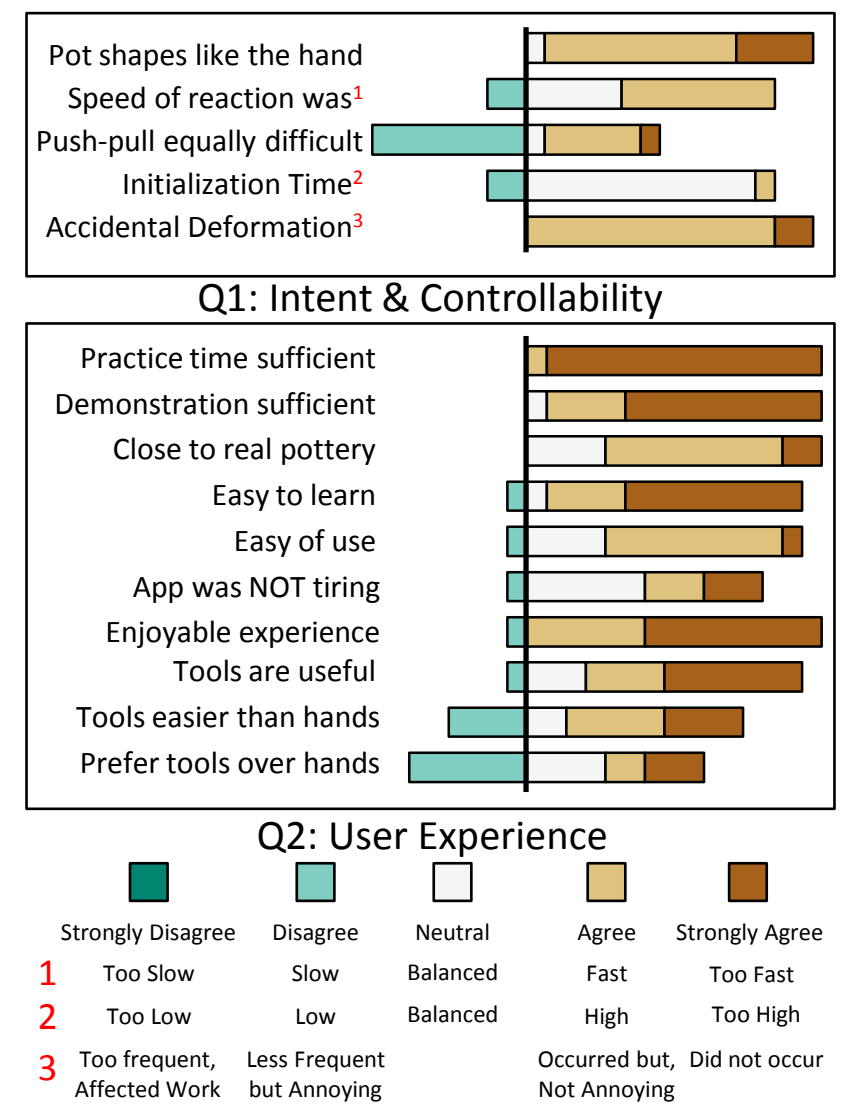

Figure 19: User response to are shown for grasp+motion. While the robustness to accidental deformations was perceived to be negligible (a), many users still perceived pulling to be difficult. Users agreed regarding the usefulness of tools but were not in general agreement about preferring them over hands.

763 provided to them and planned how to use them for shaping the ${ }_{764}$ pots. Users created 4 pots on average (max: 8, min: 2 ) within 7656.0 minutes (std: $0.8 \mathrm{~min}$ ). In contrast to the use of hands, 766 we observed exploratory behavior in users while using tools. ${ }_{767}$ Rather than creating pots, most users were more interested in 768 finding out the effect of each of the objects provided to them. 769 This explained the decrease in the average number of pots in 770 the composition task. One of the difficulties with the use of 771 hands was the inability to create thin concavities. With the use 772 of tools (Figure 18(a),(d)), users could achieve this easily. The 773 most interesting behavior that was observed was the tendency to 774 create convex deformations, which the users achieved by com775 bining two different objects, so as to simulate a grasping hand. 776 This was evident from the users' fascination with scissors (Fig777 ure 18(b)). Another important observation was the direct asso778 ciation the users made between the shape of the tool and the 779 purpose it could be used for. The motion of the hand was af780 fected by this association. For instance, while using a white781 board eraser (Figure 18(c)), the most common motion was that 782 of smoothing the pot. Similarly, for objects with grasp-like ge783 ometries, users invariably tried convex deformations by pulling 784 (Figure 18(e)). One user fashioned a new tool by combining 785 different Shapescapes ${ }^{\mathrm{TM}}$ parts. This provided the convenience 786 of holding the tool at the "handle" and deforming the pot using
787 fine hand movements (Figure 18(f)).

788 Intent \& Controllability $(Q 1)$. We see evident improvements in 789 the perception of intent recognition quality, initialization time, 790 and robustness to accidental deformations (Figure 19). How791 ever, despite the decrease in completion time (task T1) there 792 was no significant improvement in the user's perception of in793 consistency between pulling and pushing. In this case, reason 794 for this perception was primarily related to the visual and tactile 795 feedback rather than the algorithm for pulling itself. This was 796 evident from the user's comments such as: "I think the reason 797 pushing and pulling were different were because the pulling you 798 had to 2 contacts with the pot and pushing you only needed one. 799 I had a hard time understanding the depth of the pot making it 800 hard to get two contacts on the pot". One user also suggested: 801 "I think it would be better if I get some feeling when I touch the 802 pottery. It [would] make me feel more real and easier to control 803 my hand. Then it would be better to have some sounds when I 804 touch the pottery".

${ }_{805}$ User Experience (Q2). The experience was mostly positive, 806 similar to the proximal-attraction approach (Figure 19(b)). In 807 particular, users liked the use of tools and the smoothing opera808 tion the most. One user commented: "The freeform design with 809 tools was the most fun, as I could spend most of my time focus810 ing on the design aspect as opposed to focusing on minimizing 811 errors.". According to another user: "The pottery changing ac812 cording to my hand shape is so real. While smoothing, I could 813 shape it as well, I like to do it this way a little bit.".

\section{6.4. Limitations}

815 Our method is currently implemented for pottery, which is 816 essentially a one dimensional deformation. Further, we ob817 served that the use of 2D displays is a factor due to which users 818 tend to use side configurations. We believe that $3 \mathrm{D}$ visual feed819 back will encourage users to access the front and back faces. 820 One user noted: "This application with haptic feedback could 821 train people for pottery before they actually perform it". This 822 strongly indicates that the lack of tactile feedback is a critical 823 component that is missing from our current system.

824 Severe occlusion resulting from camera position and hand 825 orientation is an issue particularly for skeletal based gesture 826 recognition. We partly addressed this challenge using our PCL827 based approach which can make use of partial data even when 828 the full hand skeleton is intractable. However, occlusion is an 829 inherent problem in any camera-based method. Investigation of 830 optimal camera position and use of multiple cameras at strate831 gic locations is important. Secondly, we provided a method for 832 temporally adaptive persistence.

In our current implementation, the definition of active re${ }_{834}$ gions is in terms of $2 \mathrm{D}$ profile topology rather than actual dis835 tances in real space. Thus, our implementation is dependent on ${ }_{836}$ PCL sampling relative to the mesh resolution of the pot. Inde837 pendence from the sampling resolution may be addressed with 838 an adaptive approach wherein new sections could be added ac839 cording to manipulators or old ones removed based on geomet840 ric properties of the pot profile such as curvature. 

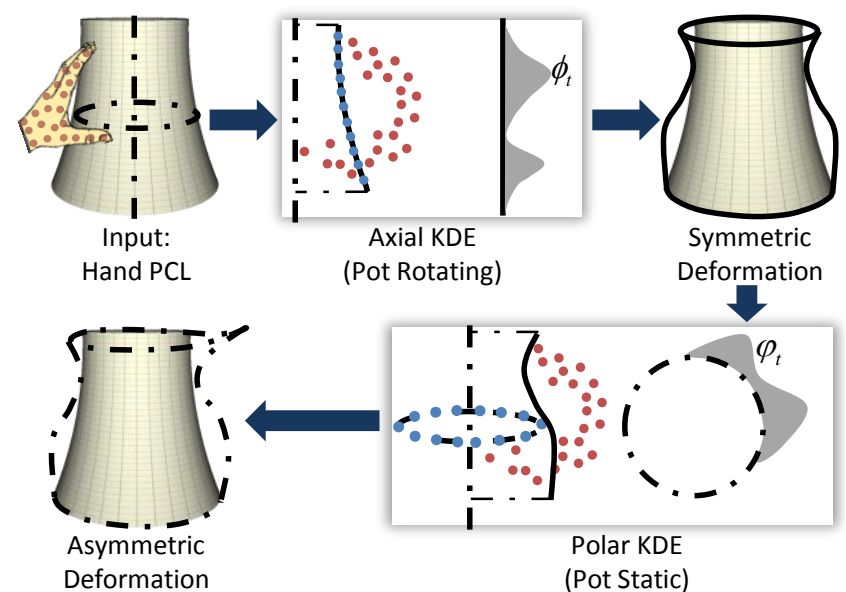

Figure 20: Asymmetric deformation can be applied to a pot in two steps. When the pot is rotating, we apply the axial KDE (top row) of the hand PCL for deforming the profile of the pot. Subsequently, users can stop rotating the pot and deform the pot locally using the polar KDE (bottom row).

In terms of our evaluation approach, our participants were 842 primarily from science and engineering background. Even though 843 some users had prior experience with creative tasks such as pot844 tery and computer-aided design, studying our approach with art 845 students would provide additional insights on user experience 846 and utility of our approach.

\section{${ }_{847}$ 7. Discussion}

\section{7.1. Spectrum of Expressiveness:}

849 One aspect that is both advantageous and disadvantageous 850 in our approach is that different users can achieve the same ${ }_{851}$ target shape using different strategies for grasping, reaching, 852 and deforming a shape. While this provides flexibility and in853 tuitiveness to the user, it also results in increasing the time 854 taken by the user to reach to a desired shape. The evalua855 tion of proximal-attraction evidently indicated that there needs 856 to be a balance between completely free-form interaction and 857 symbolic approaches. This is what we attempted through the 858 grasp+motion approach. The main advantage that our process ${ }_{859}$ provided was the discovery of relevant grasp information that 860 is useful to design continuous operations such as shape defor861 mation. Our grasp based approach can serve as a starting point 862 for designing grasp-based interactions using cleaner data such 863 as hand-skeleton [10].

\section{7.2. Definition of Intent:}

865 We began with a simple classification of intent through the 866 analogy of structuring operators inspired by Delamé's [28] work. ${ }_{867}$ However, users' description of actions and expectation strongly 868 indicates towards a richer and more complex mental model for ${ }_{869}$ deformation processes. To this effect, we had to include a third 870 class of operation, namely "smoothing" which evidently im871 proved the performance of the user. Though this aspect is not 872 new in 3D modeling in general, this aspect of refinement is cer873 tainly worth investigating from a perceptual point of view.
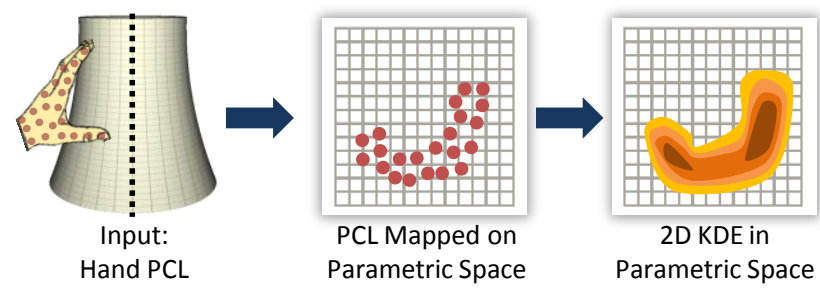

2D KDE in
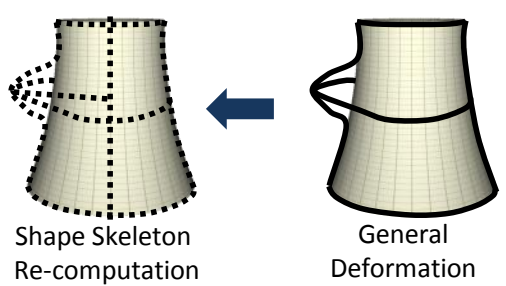

Parametric Space

Re-computation

Deformation

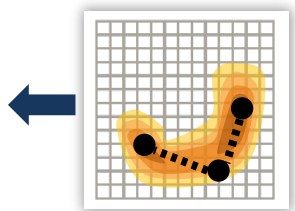

Skeleton in

Parametric Space

Figure 21: The computation of two-dimensional KDE in the parametric space of a cylindrical surface leads to the computation of grasp and motion for an arbitrary orientation of the hand PCL with respect to the surface. This allows for arbitrary ddeformation of the surface. Recomputing and segmenting the deformed surface using the method of Bærentzen et al. [33] provides a generalized deformation approach using our KDE based approach.

\section{7.3. Generalization:}

875 Although we demonstrated intent classification for rotation876 ally symmetric shapes, the general approach of computing KDE 877 to characterize grasp and motion can be extended to the defor878 mation of arbitrary shapes. Here, we propose such an extension 879 in two steps. First, we will consider asymmetric deformation 880 in the context of pottery itself. For this, we begin by noting 881 that our approach summarizes the hand grasp and motion by 882 computing a one-dimensional axial KDE of the hand PCL on 883 the pot's surface. In the same way, we can also compute the 884 one-dimensional polar KDE of the PCL (Figure 20). Thus, by 885 combining two one-dimensional KDE computations (axial and 886 polar), we can enable users to create asymmetric features on the 887 pots.

888 To see how these ideas can be used to conceptualize an arbi889 trary deformation of a shape, we make two observations. First, 890 the pot is a cylindrical shape with a simple parametric repre891 sentation and the axis of the cylinder is essentially its skeleton. 892 Thus, given the hand's PCL in an arbitrary orientation with re893 spect to the cylinder's surface, its two-dimensional KDE can be 894 computed in the parametric space as a simple means to deter895 mine the grasp and motion of the hand (Figure 21). The con896 sequent deformation of the cylinder would inevitably result in 897 the need for re-computing the skeletal structure of the surface. 898 This is where we invoke our second observation that an arbi899 trary 3D surface model can be converted to a set of connected 900 cylinders using the recent work by Bærentzen et al. [33] that 901 demonstrates the conversion of arbitrary triangle meshes into 902 polar-annular meshes (PAM). The PAM representation effec903 tively segments 3D shapes into generalized cylinders. Thus, 904 the combination of two-dimensional KDE with the PAM repre905 sentation can be used for deforming arbitrary meshes. 


\section{7.4. Precise \& Selective Reachability:}

$907 \quad$ One user aptly commented: "Sometimes it is hard to use the ${ }_{908}$ palm because it may deform the surface too much. The context 909 of barely touching does not seem too well implemented. How910 ever, if you do this very carefully you can do the barely touching ${ }_{911}$ but may make your arm tired a little.". This is the problem of 912 precise and selective reachability wherein one is required to 913 reach and manipulate a local region of an object without affect914 ing neighboring regions. There is extensive volume of work 915 that investigates distal selection, manipulation, and navigation $916[34,35,36]$ of objects. We believe that precision and selectivity 917 are problems worth investigating for close-range, i.e. proximal ${ }_{918}$ 3D manipulations in mid-air.

\section{${ }_{919}$ 8. Future Directions \& Conclusions}

920 Our first goal is to extend the grasp+motion approach for 921 arbitrary meshes. This would involve several computational 922 challenges since distance computations and KDE computation 923 would be on 2-manifolds. Secondly, we intend to study how 924 user perception ad performance is affected by adding 3D visual 925 feedback and also tactile feedback. Finally, with our approach, ${ }_{926}$ it is not possible to perform deformation using existing hand ${ }_{927}$ skeleton tracking approaches. We intend to investigate this in ${ }_{928}$ comparison to the PCL based hand representation. One key ad929 vantage of using tracked skeletons is that there is a direct corre930 spondence between the fingers and palm which can give useful 931 movement information for better intent detection. This would ${ }_{932}$ help segmenting users intentional and unintentional movements 933 [37]. One of the main observations in our preliminary explo${ }_{934}$ ration was that users from different backgrounds and age group 935 had different ways of using the pottery tool. In our future works, 936 we want to understand how experience, performance, and cre${ }_{937}$ ative outcomes will change with respect different user groups ${ }_{938}$ such as artists, engineering designers, and young participants.

939 We presented a spatial interaction technique that uses hand ${ }_{940}$ grasp and motion for intent expression in virtual pottery. This 941 approach enables a paradigm shift from existing gesture-based 942 procedural events towards non-procedural and temporally con${ }_{943}$ tinuous processes in the context of shape deformation. In other 944 words, our work enables users to achieve what they intend in 945 the way they see fit. To the best of our knowledge, no existing 946 hand-based spatial modeling scheme offers such diverse con947 texts of user input, for instance the use of everyday real objects 948 as tools for virtual shaping, with controllable outcomes. The 949 idea creates new pathways for further research exploring cre950 ative design contexts in a "what you do is what you get" frame951 work.

\section{9. Acknowledgments}

953 We thank the reviewers for their valuable feedback and com954 ments. This work was supported by the NSF-AIR Award No. 955 1312167, the NSF Award No. 1235232 from CMMI-EDI, the 956 NSF Award No. 1329979 from CPS:Synergy, and the Donald 102
${ }_{957}$ W. Feddersen Chair Professorship at the School of Mechani958 cal Engineering. Any opinions, findings, and conclusions or 959 recommendations expressed in this material are those of the au960 thor(s) and do not necessarily reflect the views of the National ${ }_{961}$ Science Foundation. We thank Dr. Raja Jasti (ZeroUI) for pro962 viding valuable suggestions and feedback on our work. We also 963 thank Kevin Lee Jr. (ZeroUI) for the artwork of our pottery in964 terface. Professor Ramani discloses he is a co-founder of Zer965 oUI that has licensed related technology.

\section{References}

[1] Segen J, Kumar S. Gesture vr: vision-based 3d hand interace for spatial interaction. In: Proceedings of the ACM international conference on Multimedia. 1998, p. 455-64.

[2] Wang R, Paris S, Popović J. 6d hands: markerless hand-tracking for computer aided design. In: Proceedings of the ACM symposium on User interface software and technology. 2011, p. 549-58.

[3] Han Y, Han Bj. Virtual pottery: a virtual $3 \mathrm{~d}$ audiovisual interface using natural hand motions. Multimedia Tools and Applications 2013;:1-17.

[4] Vinayak , Murugappan S, Piya C, Ramani K. Handy-potter: Rapid exploration of rotationally symmetric shapes through natural hand motions. Journal of Computing and Information Science in Engineering 2013;13(2).

[5] Vinayak, Murugappan S, Liu H, Ramani K. Shape-it-up: Hand gesture based creative expression of $3 \mathrm{~d}$ shapes using intelligent generalized cylinders. Computer-Aided Design 2013;45(2):277-87.

[6] Song J, Cho S, Baek SY, Lee K, Bang H. Gafinc: Gaze and finger control interface for $3 \mathrm{~d}$ model manipulation in $\{\mathrm{CAD}\}$ application. ComputerAided Design 2014;46(0):239 -45.

[7] Holz C, Wilson A. Data miming: inferring spatial object descriptions from human gesture. In: Proceedings of the ACM conference on Human factors in computing systems. 2011, p. 811-20.

[8] Leap motion sculpting. https://airspace.leapmotion.com/ apps/sculpting/windows; 2013.

[9] Iason Oikonomidis NK, Argyros A. Efficient model-based 3d tracking of hand articulations using kinect. In: Proceedings of the British Machine Vision Conference. 2011, p. 101.1-101.11.

[10] Leap motion skeletal tracking. https://developer. leapmotion.com/features; 2015.

5 [11] Vinayak , Ramani K. A gesture-free geometric approach for mid-air expression of design intent in $3 \mathrm{~d}$ virtual pottery. Computer-Aided Design 2015;69:11 - 24 .

[12] Vinayak , Ramani K. Hand grasp and motion for intent expression in mid-air virtual pottery. In: Proceedings of the 41st Graphics Interface Conference. GI '15; Canadian Information Processing Society; 2015, p. 49-57.

[13] Ren G, O'Neill E. 3d selection with freehand gesture. Computers \& Graphics 2013;37(3):101 -20.

[14] Walter R, Bailly G, Müller J. Strikeapose: Revealing mid-air gestures on public displays. In: Proceedings of the SIGCHI Conference on Human Factors in Computing Systems. CHI '13; New York, NY, USA: ACM. ISBN 978-1-4503-1899-0; 2013, p. 841-50.

[15] Karam M, Schraefel MC. A taxonomy of gestures in human computer interactions. Technical Report; University of Southampton; 2005.

10 [16] Baudel T, Beaudouin-Lafon M. Charade: Remote control of objects using free-hand gestures. Commun ACM 1993;36(7):28-35.

1012 [17] Walter R, Bailly G, Valkanova N, Müller J. Cuenesics: Using mid-air 1013 gestures to select items on interactive public displays. In: Proceedings of 1014 the 16th International Conference on Human-computer Interaction with 1015 Mobile Devices \&\#38; Services. MobileHCI '14; New York, NY, USA: 1016 ACM; 2014, p. 299-308.

[18] Quek F, McNeill D, Bryll R, Duncan S, Ma XF, Kirbas C, et al. Multimodal human discourse: Gesture and speech. ACM Trans Comput-Hum Interact 2002;9(3):171-93.

[19] Rateau H, Grisoni L, De Araujo B. Sub-space gestures. Elements of design for mid-air interaction with distant displays. Research Report RR8342; INRIA; 2013 
1023 [20] Jeannerod M. The formation of finger grip during prehension. a cortically 1024 mediated visuomotor pattern. Behavioural Brain Research 1986;19(2):99 $1025-116$.

1026 [21] Armbruster C, Spijkers W. Movement planning in prehension: do in1027 tended actions influence the initial reach and grasp movement? Motor 1028 Control 2006;10(4):311.

1029 [22] Cutkosky M. On grasp choice, grasp models, and the design of hands for 1030 manufacturing tasks. Robotics and Automation, IEEE Transactions on $1031 \quad 1989 ; 5(3): 269-79$.

1032 [23] Zheng J, De La Rosa S, Dollar A. An investigation of grasp type and 1033 frequency in daily household and machine shop tasks. In: Robotics and 1034 Automation (ICRA), 2011 IEEE International Conference on. 2011, p. 1035 4169-75.

1036 [24] Mapes DP, Moshell JM. A two handed interface for object manipulation 1037 in virtual environments. Presence 1995;4(4):403-16.

1038 [25] Boulic R, Rezzonico S, Thalmann D. Multi-finger manipulation of virtual 1039 objects. In: In Proc. of the ACM Symposium on Virtual Reality Software $1040 \quad$ and Technology (VRST '96. 1996, p. 67-74.

1041 [26] Kry PG, Pihuit A, Bernhardt A, Cani MP. Handnavigator: Hands-on 1042 interaction for desktop virtual reality. In: Proceedings of the 2008 ACM 1043 Symposium on Virtual Reality Software and Technology. VRST '08; New 1044 York, NY, USA: ACM. ISBN 978-1-59593-951-7; 2008, p. 53-60.

1045 [27] Leyton M. A process-grammar for shape. Artif Intell 1988;34(2):213-47. 1046 [28] Delamé T, Léon JC, Cani MP, Blanch R. Gesture-based design of 2d 1047 contours: An alternative to sketching? In: Proceedings of the Eighth Eu1048 rographics Symposium on Sketch-Based Interfaces and Modeling. SBIM 1049 '11; New York, NY, USA: ACM. ISBN 978-1-4503-0906-6; 2011, p. 1050 63-70

1051 [29] Holt CC. Forecasting seasonals and trends by exponentially weighted 1052 moving averages. International Journal of Forecasting 2004;20(1):5-10.

1053 [30] Stuerzlinger W, Wingrave C. The value of constraints for $3 \mathrm{~d}$ user inter1054 faces. In: Brunnett G, Coquillart S, Welch G, editors. Virtual Realities. 1055 Springer Vienna; 2011, p. 203-23.

1056 [31] Yoo JC, Han T. Fast normalized cross-correlation. Circuits, Systems and $1057 \quad$ Signal Processing 2009;28(6):819-43.

1058 [32] Rousseeuw PJ, Ruts I, Tukey JW. The bagplot: A bivariate boxplot. The $1059 \quad$ American Statistician 1999;53(4):382-7.

1060 [33] Bærentzen JA, Abdrashitov R, Singh K. Interactive shape modeling using 1061 a skeleton-mesh co-representation. ACM Trans Graph 2014;33(4):132:1$1062 \quad 132: 10$

1063 [34] Hand C. A survey of 3d interaction techniques. Computer Graphics Fo1064 rum 1997;16(5):269-81.

1065 [35] Bowman D, Hodges L. An evaluation of techniques for grabbing and 1066 manipulating remote objects in immersive virtual environments. In: Pro1067 ceedings of the ACM Symposium on Interactive 3D Graphics. 1997, p. 1068 53-8.

1069 [36] Hinckley K, Pausch R, Proffitt D, Kassell NF. Two-handed virtual 1070 manipulation. ACM Transactions on Computer-Human Interaction $1071 \quad$ 1998;5(3):260-302.

1072 [37] Choumane A, Casiez G, Grisoni L. Buttonless clicking: Intuitive select 1073 and pick-release through gesture analysis. In: Virtual Reality Conference 1074 (VR), 2010 IEEE. 2010, p. 67-70. 


\section{*Graphical Abstract}

Extracting Hand Grasp \& Motion for Intent Expression in Mid-Air Shape Deformation A Concrete \& Iterative Exploration through a Virtual Pottery Application

Vinayak, Karthik Ramani*

School of Mechanical Engineering, Purdue University

*School of Electrical and Computer Engineering, Purdue University (by courtesy)

We describe an interaction technique for mid-air virtual pottery. Given a point-cloud of the user's hand, our technique estimates grasp and motion for the pot's deformation using kernel-density estimation.

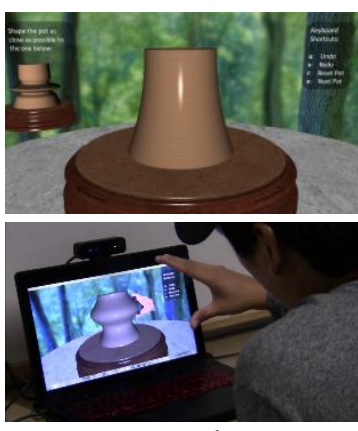

Hand
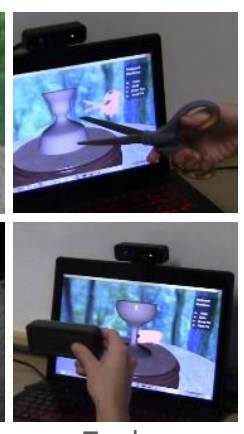

Tools
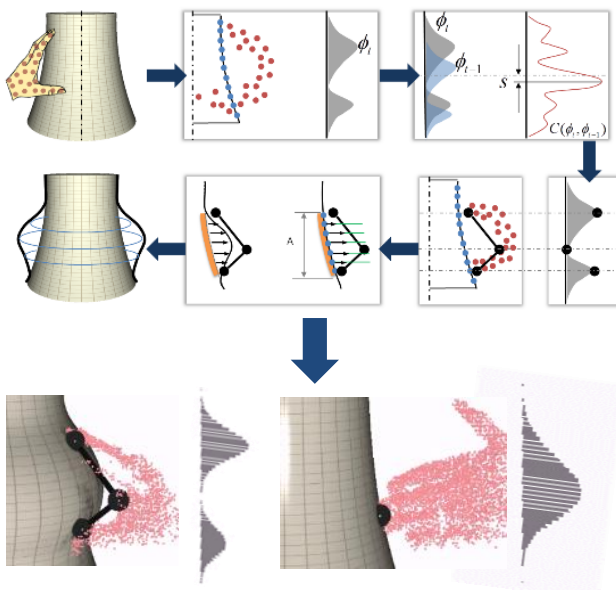

Grasp detection from Kernel density 